\title{
Al and Cultural Heritage Image Collections: Opportunities and challenges
}

Konstantina Karterouli

Dumbarton Oaks, Harvard University

$170332^{\text {nd }}$ Street NW, Washington, DC, 20007, USA

karterouli@gmail.com

\author{
Yota Batsaki \\ Dumbarton Oaks, Harvard University \\ $170332^{\text {nd }}$ Street NW, Washington, DC, \\ 20007, USA \\ batsakiy@doaks.org
}

\begin{abstract}
This paper contributes to the discussion about the opportunities and challenges of applying computer vision and machine learning to archival image collections of significant cultural heritage value. We explore these questions from an institutional perspective. Our case study is a pilot project developed at Dumbarton Oaks, a research institute and library, museum, and historic garden affiliated with Harvard University and located in Washington, DC. The project focused on a collection of 10,000 images of Syrian monuments in the institution's Image Collections and Fieldwork Archives (ICFA). Drawing on that project, as well as the broader landscape of Al-based categorisation efforts in the fields of art and architecture, we will share our insights on the potential of Al to facilitate and enhance archival image access and recording. Many of the Syrian sites in the Dumbarton Oaks collection have been inaccessible to researchers and the public for over a decade and/or have been damaged or destroyed. The pilot project, undertaken in 2019-2020, was a collaboration between Dumbarton Oaks; a commercial tech partner, ArthurAl Inc.; and a computer science research team from the University of Maryland. For Dumbarton Oaks, the primary goal was to explore whether Al can improve the speed and efficiency of sharing collections and allow for more sophisticated curation by subject experts who, thanks to automation, would be relieved of the burden of rote processing. For the technology partners, the experimental value of the project lay in the availability of a collection that could be shared open access (no privacy or copyright issues) and was focused enough to yield a domain-specific training set. The methods and techniques explored included multi-label classification, multi-task classification, unsupervised image clustering, and explainability.
\end{abstract}

Image classification. Deep learning. Convolutional neural networks. Cultural heritage. Digital humanities.

\section{INTRODUCTION}

Artificial Intelligence could prove of enormous value for archival image collections like the ones held at the Dumbarton Oaks research institute in Washington, DC. Specialised image collections offer a critical research tool, while they also hold great attraction for the public through the growing digital sharing of collections. At the same time, they are increasingly important to the preservation of cultural heritage as documents of the past condition of sites and monuments. This cultural preservation value is further augmented in the case of archaeological sites of cultural and art historical interest that are not well documented, are rendered inaccessible, or have suffered subsequent damage. Al holds the potential to expedite the processing of these vast image collections, which otherwise pose formidable challenges, especially with regard to metadata application and curated access to the content material.
In recent decades, extensive efforts have been directed toward the creation of digital resources for cultural heritage management, ranging from digitisation initiatives to the development of sophisticated, first-line response actions, such as the extraction of pertinent information from social media postings. More recently, deep learning techniques (LeCun, Bengio \& Hinton 2015), and especially convolutional neural networks (CNNs), have been applied to images of cultural heritage value to perform classification and annotation tasks that are critical to documentation (Llamas et al. 2017), as well as to facilitate public access to this content. Lamas A. et al 2021 and Palma 2019 have experimented with the building of mobile applications integrating deep learning pipelines for monument recognition. With regard to landmark architecture and monuments, Llamas et al. 2017 tested several convolutional neural networks and achieved remarkable accuracy in the classification of architectural heritage images into ten categories of architectural elements compiling a dataset 
sourced from Flickr and Wikimedia Commons images. In a study by Kumar et al., images from social media postings were used toward the identification of disaster-afflicted sites. Despite the low precision results of the predictive models, the authors noted their value in greatly reducing the manual effort involved by accurately detecting appropriate candidates for further examination by experts (Kumar et al. 2020). Amato, Falchi \& Vadicamo 2016 applied deep learning to image retrieval and visual searching in the EAGLE corpus of Classical Latin and Greek inscriptions. This proved to be a promising avenue for the searching functionality in digital archives, correctly identifying more than $90 \%$ of cases. The realm of fine art painting counts a larger number of studies that employ deep neural networks for the classification of visual elements as well as painting style, reflecting the unequal availability of high-quality open access training material (Belhi, Bouras \& Foufou 2018, p. 6, Elgammal et al. 2018, Sandoval, Pirogova \& Lech 2019). Among institutional efforts, The Metropolitan Museum of Art has announced a recent partnership with Microsoft and the Massachusetts Institute of Technology to pursue a number of Al-based use cases. These aim to enrich user experiences of the Met's Open Access artworks through the detection of hidden patterns, as well as broaden access to the museum collections through automation of the tagging process and associated cost efficiencies (Choi 2019). At the same time, Al applications are being progressively introduced in the context of libraries to achieve efficiencies around the processing of textual and visual data (Cordell 2020).

Although there is an increasing number of individual technical studies involving the use of deep learning in the processing of images of cultural heritage value, the adoption of such techniques by institutional holders is in its nascent stage. This differs from the much wider adoption and popularity of advanced deep learning techniques in other fields, such as medicine and the related area of image diagnostics (Le et al. 2017).

In 2019, Dumbarton Oaks embarked on a pilot Al project focused on an image collection of 10,000 images of Syrian monuments in the Dumbarton Oaks Image Collections and Fieldwork Archives (ICFA). The project sought to evaluate the applicability of $\mathrm{Al}$ techniques to extensive holdings of archival image and fieldwork material with the aim of identifying improvements to customary workflows. In this paper, we focus on the challenges and opportunities of the application of such mature and well-known computer vision and machine learning applications in the context of a research library, to streamline the cataloguing of vast archival collections and render them more quickly and openly accessible.

\section{AI IN THE DUMBARTON OAKS COLLECTIONS}

The pilot project sought to expedite and expand the digital cataloguing and curation of image collections in ICFA. Machine learning and computer vision techniques were applied to the processing of the newly acquired Frank Kidner collection of primarily Byzantine, but also Roman, Islamic, and prehistoric monuments in Syria. Kidner made six trips to Syria in the 1990s to document, in vivid colour photography, the architectural remains of the Dead Cities in the Belus Massif, a limestone plateau in northwestern Syria. This group of approximately seven hundred former settlements, gradually abandoned from the eighth to the tenth centuries, features well-preserved architectural remains that open a unique window to late antique rural life, the introduction of Christianity, and its influence on the architectural fabric of the region. Kidner's photographs record, over time, sites that have been inaccessible to researchers and visitors for years. Many of these sites have suffered damage or complete destruction during the Syrian civil war. The original slide collection was fully digitised upon acquisition. A sample image of the collection is shown in Figure 1. The image shows the south façade of a fifth/sixth-century domestic building in Deir Sunbul, Syria, in the presence of people. The building is no longer extant.

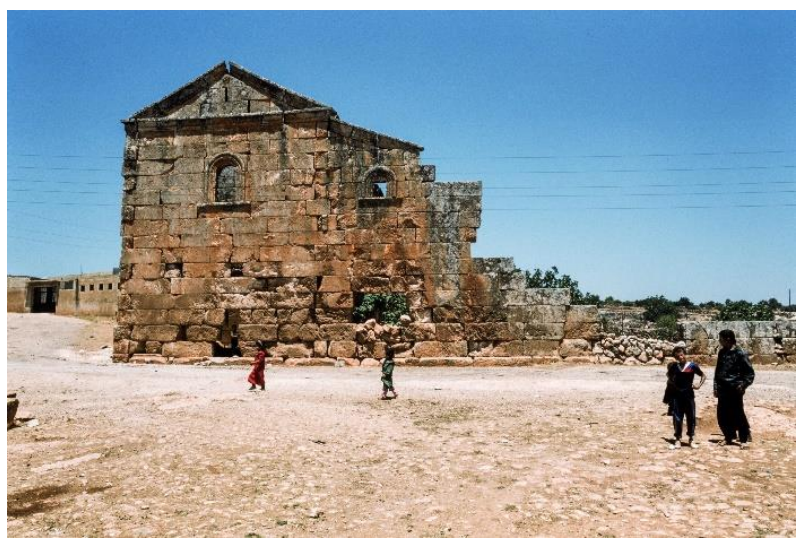

Figure 1: Deir Sunbul (Syria), south façade of west building of House 1, Dumbarton Oaks, Trustees for Harvard University, Washington, DC.

Since the priorities relating to the processing of archival collections are highly dependent on institutional context, we will describe briefly the Dumbarton Oaks collection and its context. Dumbarton Oaks is a research institute, library, museum, and historic garden affiliated with Harvard University and located in Washington, DC. The research institute supports scholarship 
internationally in the fields of Byzantine, PreColumbian, and Garden and Landscape Studies. Dumbarton Oaks welcomes around 25 residential fellows every year, who come to study the collections and advance a significant research project. The institute also promotes scholarship through annual conferences and scholarly publications. In addition to its role as an institute of advanced study, Dumbarton Oaks welcomes the public to its museum, garden, and events, and has embarked on an Access Initiative to facilitate access to its collections and resources for the broadest possible audience. Open digital access to collections and publications is a significant component of this endeavor. Dumbarton Oaks therefore has a dual mission and a dual call to excellence, in addressing its core scholarly constituencies around the world, as well as the public. This dual imperative also translates into the processing and sharing of the image collections, but providing access poses significant challenges.

The ICFA collections are part of the Research Library. The holdings relate mostly to the art and archaeology of the eastern Mediterranean and the civilizations of Mesoamerica and the Andes prior to the conquest. The collections number over a million unique items in a variety of media, from B\&W prints and slides to field notes and architectural drawings. Many of the collections have unique historical significance; for example, extensive documentation of conservation work undertaken for the Hagia Sophia monument in Istanbul by the Byzantine Institute in the 1930s. Others, such as the Kidner collection, are more recent but of no less value for cultural preservation. Despite concerted efforts by teams of staff and researchers at various career stages over the past decade, only a fraction of our holdings are catalogued and accessible through Harvard Hollis, Wikimedia Commons, and the Internet Archive. As a result, we are exploring ways to expedite the sharing of our collections without sacrificing quality.

The pace of digital processing and sharing is a particular challenge for collections that seek to articulate their contemporary relevance and move nimbly to engage with current issues and debates. When we embarked on the project, the Syrian Civil War, both as a humanitarian catastrophe and a cultural heritage disaster, were prominent in the public consciousness. One of the drivers of the application of Al technology was the hope that we might process and make openly accessible to the public and scholars alike a collection of timely content and urgent interest. Adequately annotated datasets enhance the visibility of monuments, which can be especially important for underrepresented cultures, leading to a boost of their social and cultural impact (Belhi, Bouras \&
Foufou 2018 , p. 2) while alerting the public to the need for their preservation. At the beginning of the pilot, we had created detailed metadata for less than a third of the collection. Taking into account the accelerated rate of cataloguing in the life cycle of a project, we estimated that the collection would take three years to catalogue and share with the public. For projects that aim at contemporary relevance as well as enduring value, such lengthy timeframes are a challenge. In this case, the COVID-19 pandemic struck, the Syrian Civil War sadly retreated from the public consciousness, and the urgency of cultural preservation has taken a back seat to pressing public health concerns.
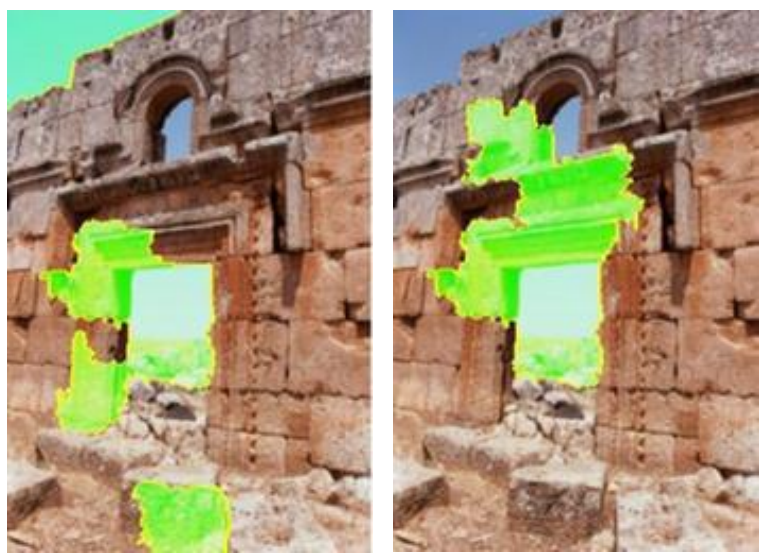

Figure 2: Explainability heatmaps for predictions of the classes "architecture" (left) and "façades" (right) by the Phase 1 classifier. Dar Qita (Syria), Church of Saint Sergios, west façade portal. Dumbarton Oaks, Trustees for Harvard University, Washington, DC.

At the same time, cultural institutions that are stewarding important collections have very long horizons, and our aim was to draw long-term lessons from the Kidner experiment. Specifically, the Al pilot served as a proof of concept for two use cases: first, the automated annotation of images with a set of subject terms that included architectural elements, building types, masonry typologies, and characterisation of image views, to serve as keyword tags; second, the automated grouping of images based on visual similarity to aid collection content organisation. A convolutional neural network classification model was developed in the first phase of the project. This model took advantage of transfer learning. Explainability techniques were explored to provide insight into the workings of the model (Figure 2). A second phase involved further exploration of classification models, including the development of a multi-task learning model, as well as the exploration of unsupervised clustering techniques for image organisation into meaningful groups. We have obtained promising results in terms of accuracy, which may validate the applicability of such models to achieve efficiencies in the context of archival image collections, and we 
are currently in the process of finalising our findings.

\subsection{Opportunities}

Our experimentation with these deep learning and computer vision techniques revealed significant opportunities but also substantial challenges in the prospective adoption of $\mathrm{Al}$ models in the context of library and archival image collections.

A key advantage of image collections for machine learning tasks is the availability of extensive image data that are curated and subject-specific. Deep learning offers well-suited applications facilitated by the concerted digitisation efforts of the last decades (Palma 2019, pp. 551-552, Yang et al. 2019). Kumar et al. 2020 note the difficulty of obtaining reliable data for the training of neural networks. Specifically, they note the diversity and noise that is typical of social media as an image source. When compared with other image datasets, collectionderived datasets can be a great candidate for classification models. This data includes, beyond the images themselves, extensive annotation metadata that can serve as training data. Especially for classification tasks, a large number of correctly labelled data is required. Models can be trained on field-specific datasets, such as the Kidner Syria dataset, directly catering to the needs of specialised collections, while at the same time expanding on standard training sets (such as ImageNet), creating new opportunities for technical experimentation.

The library cataloguing practice of using ontologies in the annotation of items can be directly exploited for machine learning tasks. Mirachi et al. 2020 emphasise the apt use of machine learning on both sides of the workflow in fields that make use of ontologies:

Due to the fact that ontologies model even complex domains, and that their creation and filling require a lot of effort, Machine Learning has been successfully applied to (semiautomated) ontology learning, alignment and annotation, and even duplicate recognition... Conversely, as clean and structured data are often required by $\mathrm{ML}$ algorithms, ontologies have been repeatedly utilised as input, given their capability to represent facts, rules and relations and point out discrepancies. (Mirachi et al. 2020, p. 315)

Data ontologies capture important expert domain knowledge that is of value to model training. Further, the opportunities provided by the data held in archives may translate into increased productivity but also, in certain scenarios, improved performance. In our models, we observed instances where the machine outperformed human cataloguing, facilitating regularisation in metadata curation (Llamas et al. 2017, p. 19). This can be especially helpful in situations where metadata harking back to legacy content management systems is incomplete or where a variety of curatorial approaches in legacy data may have led to silos of information and resulting missing connections among artworks and buildings.

Importantly, the metadata that accompany archival image collections is not restricted to data that can serve as labels to train visual features, but can also offer contextual labels to serve multi-task models. For example, Belhi, Bouras \& Foufou 2018 proposed a multi-modal classification approach based on a multi-task neural network where a convolutional neural network was designed for visual feature learning and a regular neural network performed textual feature learning. In this case, the authors took as input the image, the style, the medium, and the genre of a given painting, with the model trained to predict the painting's artist and year of creation.

Finally, as deep learning neural networks learn to identify patterns and features directly from the data, there is the potential for correlations that can prompt new research questions. As an institute for advanced study we place high value on the research potential of Al-processed image collections. Explainability techniques, in particular, could prove most promising for the detection of hidden relations among monuments and artworks, raising interesting new questions around crosscultural exchange and formation processes attached to cultural production.

\subsection{Challenges}

The project also posed a number of challenges that fall into three broad areas. These concern the requirement for significant subject expertise throughout the dataset building and model evaluation; the relationship and trade-off between task automation and human effort; and adapting data curation workflows to different tasks and goals.

Specifically, subject expertise was required at several stages of the project:

(i) Curation of the training and test sets for the classification use case required intimate familiarity with the large set of unlabeled images and the class definitions in place

(ii) Computer vision annotation required the comprehensive manual labell 
(iii) ing of the extensive training set, despite the existence of available metadata

(iv) Qualitative review was needed at various points, including the evaluation of the classification and clustering model predictions, additional labelling for a small number of classes that performed poorly, and in the selection of additional images from adjacent collections to add to the training data for classification

While digitised image archives are uniquely positioned to support the training of machine learning models due to their ample and often structured metadata, the available data requires assessment to ensure its suitability for computer vision applications. The creation of balanced training datasets that offer adequate and representative examples of the output classes, while facilitated by the nature of collections, (which tend to be homogenous by design), still requires significant pre-processing time. A plethora of data considerations needs to be addressed. For example, variations or inconsistencies in working class definitions and differing class applications across a variety of legacy data and different cataloguers and collections can result in significant noise in the data. (Despite the adoption of term vocabularies, term application may differ materially). Challenges may also arise from the quality of archival images or the presence of text in the image field (Cordell 2020, pp. 22-23).

Thus, beyond the requirement for subject expertise, the relationship between task automation and human effort needs to be considered when embarking on Al projects. The initial investment needed for an Al application is high. This frontloaded effort suggests that Al projects in domainspecific collections make more sense for institutions with adjacent collections and/or when there is a potential for institutional collaboration. The original investment in clean and comprehensive metadata is an argument in support of metadata sharing and collaboration among institutions, providing the opportunity to share in publicly available domain-specific models and training data that can compensate for areas of weak institutional holdings (Cordell 2020, pp. 5253). Further, as a one-off project, this was a stimulating and promising experiment for Dumbarton Oaks but did not translate easily into expedited cataloguing workflows. Additional investment of resources is required; for example, the development of integrations that will allow for a more seamless inclusion of the model results into the traditional workflows, as well as interfaces that may offer alternative types of image access for the public. Such interfaces can enrich the user experience, while potentially circumventing the need to abide by established content management systems that may not afford the required flexibility to integrate with machine learning work pipelines.

Last but not least, adaptive and flexible approaches to data curation may be best suited to the effective adoption of Al-based projects, especially in the following areas:

(i) How we think about metadata creation and curation, most notably the difference between labeling needed for computer vision training versus library cataloguing and the setting of clear priorities for achieving the right $\mathrm{Al}$ targets, e.g. opting for classification models aimed for curation (precision) versus models for opening up new research questions (recall)

(ii) Communication between library professionals, subject experts, and technology partners, as it pertains to the need for a common vocabulary, the challenge of ongoing coordination, and the need to establish clear shared goals at the beginning

(iii) Consideration of evaluation metrics, since for $\mathrm{Al}$ initiatives to be successful, there may be a need to embrace non-perfection and an active, iterative process of constant improvement

(iv) Evolving and reinforced project teams, including the introduction of new roles and relevant training resources

\subsection{Future directions}

The Dumbarton Oaks Al pilot established our interest in the application of Al to our extensive image collections. A next step in scaling our Al efforts may be the evaluation of the best performing models for potential use in adjacent collections. Other promising directions are the development of a pipeline and platform to host developed models, to allow for more efficient model training and finetuning, and better integration into our cataloguing workflow; as well as a website interface for direct user access to model results. 


\section{REFERENCES}

Amato G., Falchi F., and Vadicamo L. (2016). Visual Recognition of Ancient Inscriptions Using Convolutional Neural Network and Fisher Vector. Journal on Computing and Cultural Heritage, 9(4), 21.

Belhi, A., Bouras, A., and S. Foufou (2018) Leveraging Known Data for Missing Label Prediction in Cultural Heritage Context. Applied Sciences, 8(10), 1768.

Choi J. (2019) Exploring Art with Open Access and Al: What's Next?

https://www.metmuseum.org/blogs/now-at-themet/2019/met-microsoft-mit-exploring-art-openaccess-ai-whats-next (retrieved 13 March 2021).

Cordell R. (2020) Machine Learning + Libraries: A Report on the State of the Field. Library of Congress, Washington, D.C.

Elgammal A., Mazzone M., Liu B., Kim D., and Elhoseiny M. (2018) The Shape of Art History in the Eyes of the Machine. Proceedings of the ThirtySecond AAAI Conference on Artificial Intelligence, New Orleans, Louisiana, USA, February 2-7, 2018. AAAI Press, New Orleans.

Kumar, P., Ofli F., Imran M., and Castillo C. (2020) Detection of Disaster-Affected Cultural Heritage Sites from Social Media Images Using Deep Learning Techniques. Journal on Computing and Cultural Heritage, 13(3), 23.

Lamas A., Tabik S., Cruz P., Montes R., MartinezSevilla A., Cruz T., and Herrera F. (2021) MonuMAI: Dataset, Deep Learning Pipeline and Citizen Science Based App for Monumental Heritage Taxonomy and Classification. Neurocomputing, 420, pp. 266-80.
Le L., Zheng Y., Carneiro G., and Yang L. (eds.) (2017). Deep Learning and Convolutional Neural Networks for Medical Image Computing: Precision Medicine, High Performance and Large-Scale Datasets. Springer International Publishing, Cham.

LeCun Y., Bengio Y., and Hinton G. (2015) Deep Learning. Nature, 521 (7553), pp. 436-444.

Llamas, J., Lerones P. M., Medina R., Zalama E. and Gómez-García-Bermejo J. (2017) Classification of Architectural Heritage Images Using Deep Learning Techniques. Applied Sciences, 7, 992.

Mirachi C., Di Martino, B., Pavan A., and Esposito, A. (2020) Impact of Industry 4.0 in Architecture and Cultural Heritage: Artificial Intelligence and Semantic Web Technologies to Empower Interoperability and Data Usage. In Bolognesi C. M. and Santagati C. (eds.), Impact of Industry 4.0 on Architecture and Cultural Heritage. IGI Global, Hershey.

Palma, V. (2019) Towards Deep Learning for Architecture: A Monument Recognition Mobile App. The International Archives of the Photogrammetry, Remote Sensing and Spatial Information Sciences (XLII-2/W9 2019), Bergamo, Italy, 6-8 February 2019, 551-556. Bergamo.

Sandoval C., Pirogova E., and Lech M. (2019). Two-Stage Deep Learning Approach to the Classification of Fine-Art Paintings. IEEE Access, 7, pp. 41770-41781.

Yang S., Mook Oh., Merchant D., Howe B., and West. J. (2019) Classifying Digitized Art Type and Time Period. Proceedings of International Journal for Digital Art History (DSDAH 2018), London, UK, August 2018. London. 